\title{
APPLICATION OF TEXTURE FEATURES AND MACHINE LEARNING METHODS TO GRAIN SEGMENTATION IN ROCK MATERIAL IMAGES
}

\author{
KAROLINA NurZYNSKA ${ }^{凶, 1}$ AND SEBASTIAn IwASZENKO ${ }^{2}$ \\ ${ }^{1}$ Institute of Informatics, Silesian University of Technology, Gliwice, Poland, ${ }^{2}$ Central Mining Institute, \\ Katowice, Poland \\ e-mail: Karolina.Nurzynska@polsl.pl, siwaszenko@gig.eu \\ (Received May 19, 2019; revised March 6, 2020; accepted March 23, 2020)
}

\begin{abstract}
The segmentation of rock grains on images depicting bulk rock materials is considered. The rocks' material images are transformed by selected texture operators, to obtain a set of features describing them. The first order features, second-order features, run-length matrix, grey tone difference matrix, and Laws' energies are used for this purpose. The features are classified using k-nearest neighbours, support vector machines, and artificial neural networks classifiers. The results show that the border of rocks grains can be determined with above $75 \%$ accuracy. The multi-texture approach was also investigated, leading to an increase in accuracy to over $79 \%$ for the early-fusion of features. Attempts were made to reduce feature space dimensionality by manually picking features as well as by the use of principal component analysis. The outcomes showed a significant decrease in accuracy. The obtained results have been visually compared with the ground truth. The compliance observed can be considered to be satisfactory.
\end{abstract}

Keywords: classification, grain sizes, object segmentation, texture features.

\section{INTRODUCTION}

Despite great efforts, modern civilisation is still based on the efficiency of the extraction and processing of raw materials. Energy production, civil engineering, industrial manufacturing and high-tech equipment production are just a few examples of sectors whose existence would not be possible without them. However, before raw materials can be transformed into valuable goods, several technological processes have to be applied. Many of them are sensitive to rock grain size distribution and properties (Hulthén and Evertsson, 2009; Tessier et al., 2007). This influences the effectiveness of the enrichment process, machinery wear and often it is crucial for specific applications (e.g. aggregates used in civil engineering). The rock size distribution is also sometimes regulated by legal requirements, as in the fossil fuel industry. The standard methods, commonly used in the raw material industry, are labour and time-consuming. Moreover, it has already been shown that using computer-aided sorting systems is beneficial (Latała and Wojnar, 2001). Therefore, attempts are being made to develop methods and technologies enabling the determination of, at least, rock grain sizes in an automated, fast and cost-effective way. Computer vision is one of the most promising approaches being studied. However, before computer vision methods can be successfully and reliably used in an industrial environment, several problems have to be solved.
The most basic is the appropriate segmentation of grain borders and interiors. Once this has been completed, the rock grains can be counted, their shape parameters calculated, and the distribution of grain sizes designated.

It turns out, that the task of rock delineation is not straightforward, as rocks have a complex structure, are composed of multicoloured minerals, have internal edges, etc. The perfect method for the segmentation of the rock grain interior and boundary should be able to discriminate between the rock edges without being misled by the rock grain's internal structure. Such a method has not been proposed to date. In addition, other difficulties arise during the determination of rock grain sizes as the image is a $2 \mathrm{D}$ view of a $3 \mathrm{D}$ shape where the projection of the rock grains usually overlap.

There are few main pathways in which computer vision and image processing are used in rock characterisation. First of all, researchers focus on the internal structure and properties of the rock material. The optical or scanning electron microscope images are used for the identification of minerals and the internal structure of rock material. The analysis of features of mineral grains contained in the rock material is helpful in the determination of geological processes which shaped the properties of the rock material (Ailleres et al., 1995). It is also helpful in the description of the origins of a material sample (Mertens and Elsen, 2006). Image 
processing of micro-images of minerals is also used in its composition assessment (Alpana and Mohapatra, 2015; 2016; Lu et al., 2009; Lumbreras and Serrat, 1996; Mlynarczuk et al., 2014). The methods applied for image processing and analysis range from simple thresholding (Ailleres et al., 1995; Haines et al., 2015; Wang, 2008), through morphological operations (Lumbreras and Serrat, 1996; Mertens and Elsen, 2006; Mlynarczuk et al., 2014; Wang, 2008; Outal and Beucher, 2009), to texture analysis and statistical methods (Alpana and Mohapatra, 2015; 2016). The most commonly used approach for grain segmentation is the watershed algorithm.

One of the most promising though difficult applications of computer vision in rock characterisation is the determination of rock grain sizes and shapes. There are some aspects which form the scope of research: the individual grain shape and size detection, the investigations on correspondence between image based and traditionally measured grain sizes and the classification of rock material and segmentation of bulk raw materials (e.g. on a conveyor belt). The rock segmentation in the assessment of individual grain sizes and shapes is usually simplified by an image acquisition technique. Pictures are taken in a way that ensures high contrast between the stones and the background. The material is placed so that adjacent stones do not touch each other. There are a few methods mentioned in the literature to meet these requirements. A special stand with artificial lighting was used to photograph rocks placed separately on a white background (Kwan et al., 1999; Mora et al., 1998). The lighting was designed to minimise the shadow cast by the rocks. There were also examples of rocks imaged one by one on a special stand (AlThyabat and Miles, 2006). The other option here was to use a backlight to the rock particles (Al-Thyabat and Miles, 2006; Zhang et al., 2013). The modification of this method, by usage of a flat scanner, was reported by Igathinathane et al. (2012); Igathinathane and Ulusoy (2016).

The standard approach used for grain size determination is performed by sieving the sample taken from the bulk material through a set of screens. The material granulometry is characterised by the sizes of the net in the screens. There were attempts to find the relationship between the image analysis and standard method results (Zhang et al., 2013).

The problem was also investigated by Outal et al. (2008). A map function was proposed for transforming the histogram obtained from image analysis into a histogram corresponding to the results of the sieve analysis. A comparison of the obtained results with the results of the actual sieve analysis showed satisfactory compliance, provided that the buckets adopted for determining the histograms were large enough. In engineering practice, this is not a significant limitation. In the Outal et al. (2009) the power function is determined for transforming area size distribution obtained from image analysis into the 3D distribution determined by sieve analysis. Regardless of the good results presented in the above papers, one should be aware that there are many potential sources of errors. The improper delineation, insufficient image resolution, particle overlapping, etc., can influence the final result of the estimation of grain sizes in the image (Thurley, 2013). The shape characteristics of rock materials were investigated by Wang (2006), where 18 classes of grain shapes were proposed. Other methods include Ferret and equivalent diameter as a parameter which corresponds well with the results obtained by sieving (Al-Thyabat and Miles, 2006).

Though the described methods of image acquisition are almost not applicable in industrial conditions (though the interesting solution was proposed by Wang (1999)), the presented image analysis forms the basis for the use of computerised methods in mineral processing. When the problem of rock segmentation is solved, the size and shape determination methods can be applied. However, rock segmentation in images depicting the bulk material is not a trivial task. The simplest approaches concentrate on thresholding and watershed segmentation (Guyot et al., 2004; Kemeny et al., 2002; Siddiqui et al., 2010). These methods are also the basis for a few commercial software solutions (Guyot et al., 2004; Maerz, 1998; Siddiqui et al., 2010). Unfortunately, straight adoption of watershed leads to significant over-segmentation. The shapes and sizes of rock grains are barely preserved. The most sophisticated techniques use advanced pre-processing before the watershed algorithm is applied (Chatterjee and Bhattacherjee, 2011; Perez et al., 2011; Zhang et al., 2013). The pre-processing is mainly aimed at the selection of reasonable watershed starting zones. The obtained results are significantly better than those from previous attempts. Particularly, Perez et al. (2011) and Zhang et al. (2013) achieved reasonably good segmentation. However, the problems with appropriate grain edge detection as well as oversegmentation are still present. The reported research result is also based on a small set of analysed images. Some researchers concentrated on rock material characterisation (e.g. detecting the ore type) without segmenting every single grain (Ko and Shang, 2011).

The interesting research on the determination of appropriate markers for the watershed algorithm for rock grain delineation problem was presented in the 
(Outal and Beucher, 2009) and (Outal, 2006). The key concept is to find the method for marking each of the rock grain in the image. After the set of markers is established, the application of one of the segmentation algorithms could lead to proper grains boundary identifications. The proposed method uses a modifiation of ultimate opening morphological transformation. The method has been completely developed and described. Its application to rock material images allows for good grain delineation.

Some other research used texture analysis - greylevel co-occurrence matrix or wavelets (Murtagh et al., 2005; Perez et al., 2011). The use of images obtained using laser scanning was also considered (Thurley, 2013). However, even using such technology does not resolve all the problems which are encountered with the conventional approach - e.g., the images obtained from laser scanning still need segmentation. Though the improvements were clearly visible, the problem is still far from being solved. Therefore, undertaking research related to segmentation is fully justified.

This work concentrates on the segmentation of rock grains in bulk material. The application of texture feature maps, previously used in medical image analysis (Obuchowicz et al., 2018), were proposed. The features use simple first order statistics as well as multiscale, differential ones. A similar approach was also exploited for fault detection in metallic images by Cord et al. (2010). The selected classifiers were applied for differentiation between grain edges and grain interiors. The ground truth was prepared as a set of images manually segmented by experts. The 'Materials and Methods' section describes in detail the image database, the texture operators applied for feature calculation and proposed classification methods. In the 'Results' section results are presented and their importance is discussed in the 'Discussion' section. The 'Conclusions' section summarises the paper.

\section{MATERIALS AND METHODS}

The grains presented in the images are segmented by a classifier, which bases the decision on information derived from texture information. Several classification techniques are considered. Their details are presented in the 'Classification' section. The descriptive information used to train the classifier is derived from texture operators, which are implemented in such a way to return a value for each pixel in the image, instead of one value per image, as it is used in standard applications. The 'Texture operators' section presents the necessary details. Passing the image through the classifier, one achieves a segmentation map whose quality is evaluated. Finally, the details concerning data acquisition for the experiments are given in the 'Image acquisition' section.

\section{TEXTURE OPERATORS}

Texture operators perform a calculation using information conveyed in the distribution of image intensities supported by their spatial information. A wide variety of approaches exist which derive features from image texture. In this research, the first order features, FOF, second-order features, COM, run-length matrix, RLM, grey-tone difference matrix, GTDM, and Laws' energies provide interesting texture feature maps for further processing. In contrast to local binary patterns (Ojala et al., 2002) and local phase quantisation methods which were inapplicable. Pixel values in those maps were obtained using a sliding window approach (with a resolution of $21 \times$ 21 pixels) for all methods except Laws' energies - whose definition applies $5 \times 5$ pixel kernels. In the case of LAWS, the method definition determines the resolution of the sliding window. For all other techniques, the smallest possible resolution was chosen that assures the number of sampled pixels is sufficient in order to make the further calculation statistically significant. Applying a smaller window would probably make the techniques better describe the border, yet since the parameters would be calculated from very small number of samples, they would be unstable.

\section{First order features}

FOF describes image content concentrating on the knowledge derived from intensities, $i$, and distribution in the image, I. As a starting point a formalised histogram of grey-levels is calculated:

$$
h(i)=\frac{1}{W \cdot H} \sum_{x=1}^{W} \sum_{y=1}^{H} \begin{cases}1 & I(x, y)=i \\ 0 & \text { otherwise }\end{cases}
$$

where $W$ and $H$ are image width and height and $(x, y)$ are the pixel coordinates. From this structure the following standard statistical measures are derived (Materka and Strzelecki, 1998): mean, variance, skewness, kurtosis, energy, and entropy. The visualisation of these features is presented in Fig. 1. One can see that the main borders are visible in the maps prepared by each feature. However, in the case of short and less pronounced borders, the results vary. 


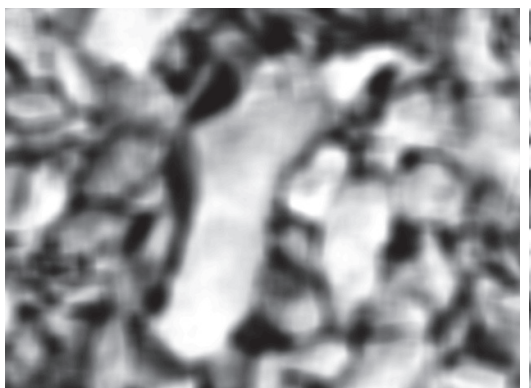

(a) Mean

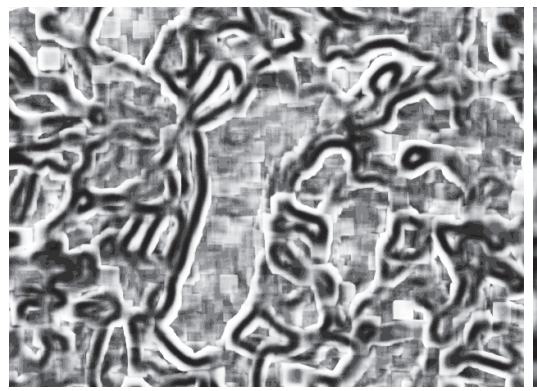

(d) Kurtosis

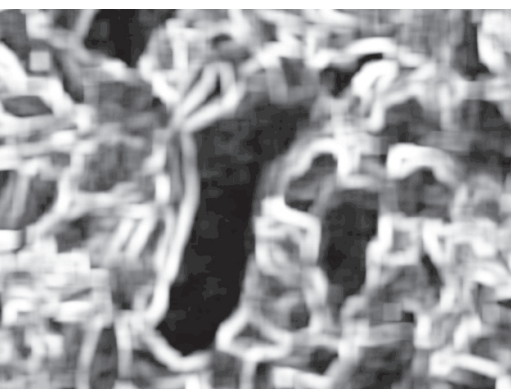

(b) Variance

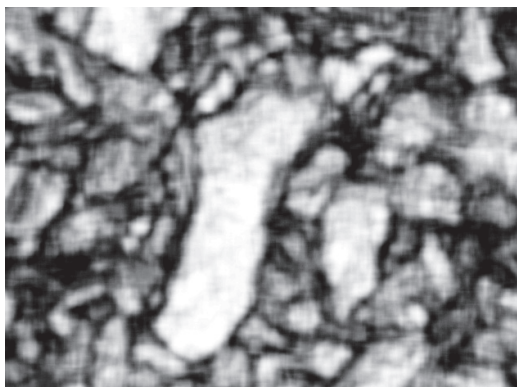

(e) Energy

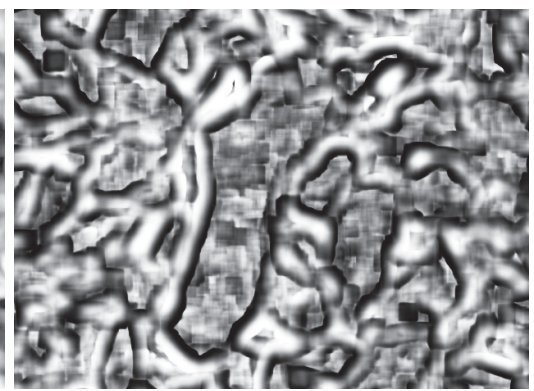

(c) Skewness

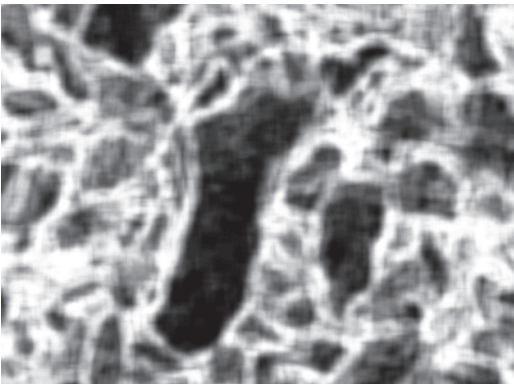

(f) Entropy

Fig. 1. FOF: visualisation of texture feature maps.

\section{Second order features}

COM was introduced by Haralick et al. (1973), where the authors described the grey tone cooccurrence matrix which keeps information on the distribution of pixel intensities and also their spatial relation. The matrix size corresponds to the total number of intensities and each entry stores the number of occurrences of pair of pixels in a distance $d$ which intensities index the matrix $(d=1$ in presented experiments). In order to remove a rotation invariance, it is suggested that matrices should be prepared for each angle from 0, 45, 90, and 135 degrees. Additionally, 14 features derived from the greytone co-occurrence matrix were used, their detailed description is given by Haralick et al. (1973), while Fig. 2 presents the outputs. Here, however, the maps are different, as the main borders which delineate big objects are clearly visible, while the distinction of weaker borders differs depending on the feature. Moreover, due to the lower computational cost, the grey-scale range was changed to 64 intensity levels.

\section{Grey-tone difference matrix}

Another approach for texture description, aimed at creating such metrics that confirm the way humans see a texture, was successfully designed as the features of a grey-tone difference matrix (GTDM) by Amadasun and King (1989). For a grey-scale image, the absolute differences between pairs of grey-level values and the average intensity of its neighbourhood $\left(\bar{I}_{x, y}\right)$ are stored in a matrix:

$$
s(i)=\sum_{x=1}^{W} \sum_{y=1}^{H}\left|I(x, y)-\bar{I}_{x, y}\right|,
$$

which is then used to derive the following texture descriptors: coarseness, contrast, business, complexity, and strength. Fig. 3 presents visualisation of the computed features. Similarly, as previously mentioned, the delineation of weak borders differs and becomes blurred.

\section{Run length matrix}

There was also an idea to describe texture based on the information concentrated in the sequences of pixels with the same pixel intensities (runs). It was observed that long runs are characteristic for coarse textures, while short runs correspond to images of good quality. The original paper about RLM introduced 5 features (Galloway, 1975), but this method was later significantly developed (Tang, 1998). Generally, the run length matrix describes the relationship between the pixel intensities and the lengths of sequences of similar values, thus counting the number of occurrence of run-length for the considered intensities. Usually, for calculation purposes, the number of intensities is quantised (to 32 colours in the presented experiments) and runs up to some value are distinguished (10 pixels in the presented case). Additionally, directions for 0 and 90 degrees were applied in the computation to diminish the rotation influence. The exemplary texture 


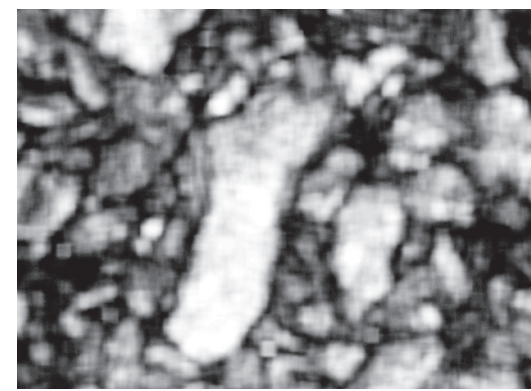

(a) Energy

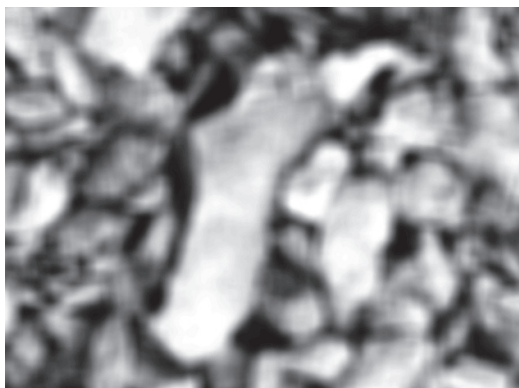

(d) Variance

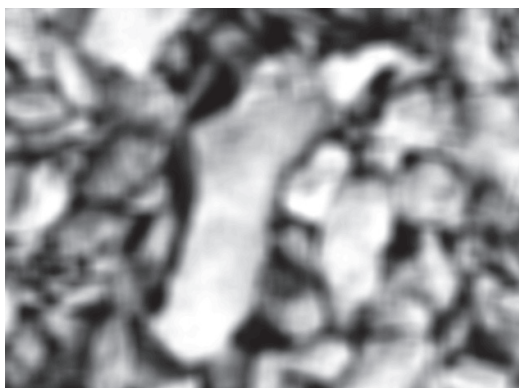

(g) Sum Variance

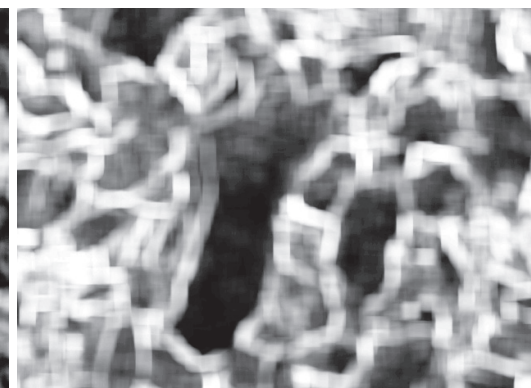

(b) Contrast

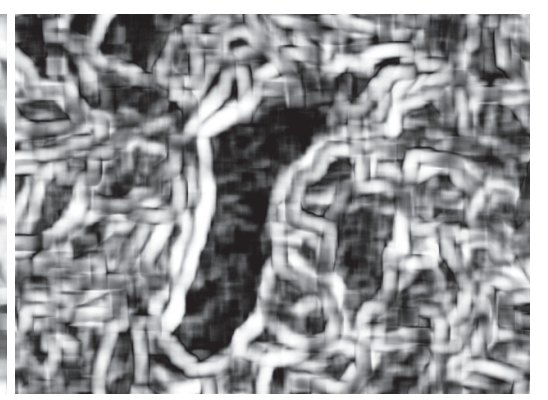

(c) Correlation

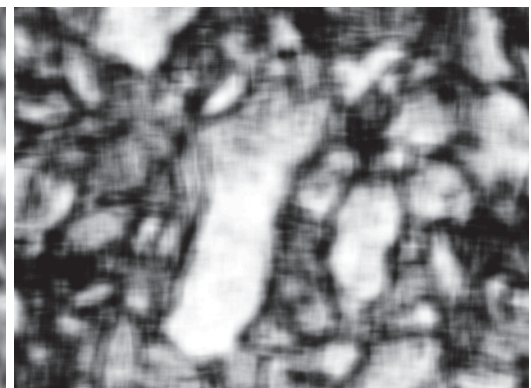

(e) Homogeneity

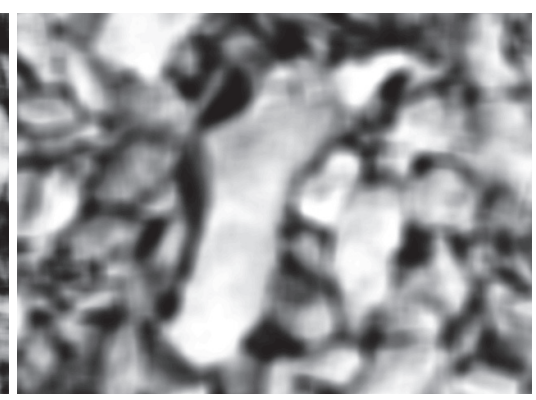

(f) Sum Average

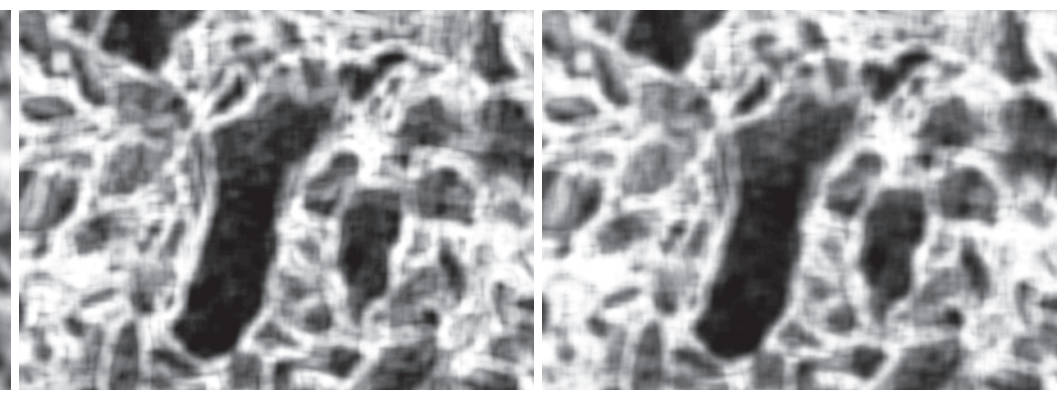

(h) Sum Entropy

(i) Entropy

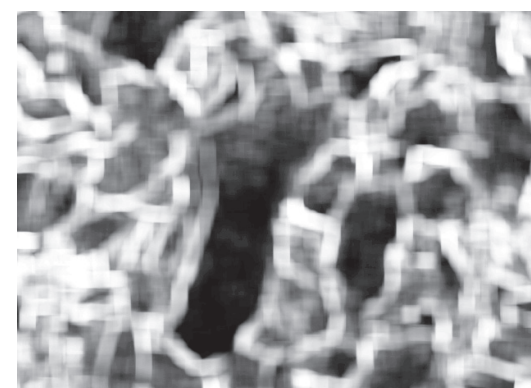

(j) Difference Variance

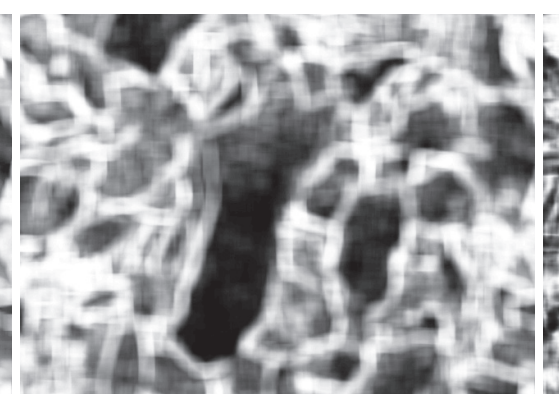

(k) Difference Entropy

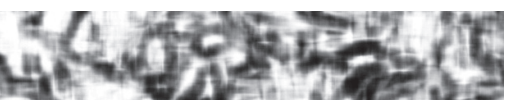

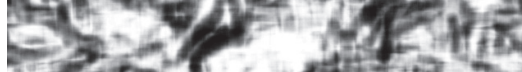

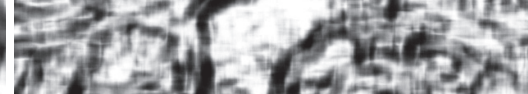

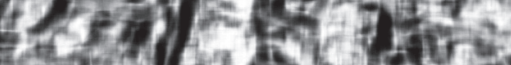

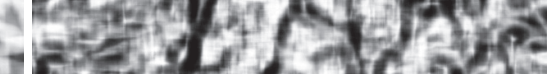

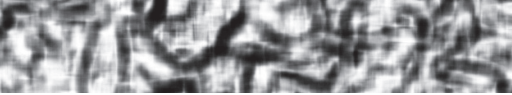

(1) Information Measure of Correlation I

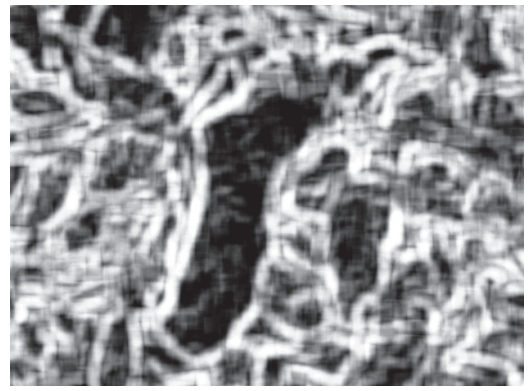

(m) Information Measure of Correlation II

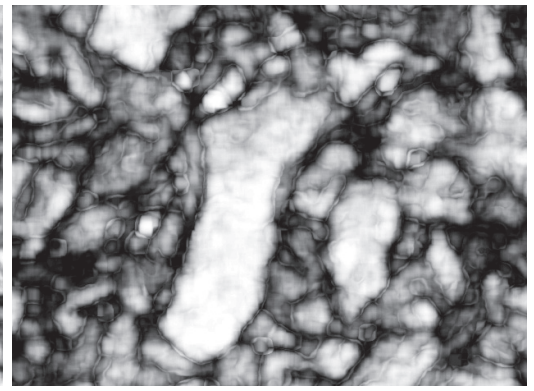

(n) Maximal Correlation Coefficient

Fig. 2. COM: visualisation of texture feature maps. 


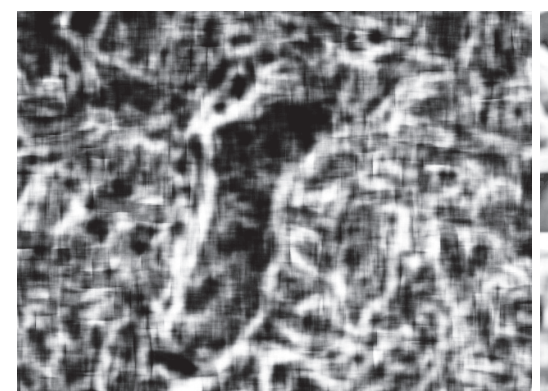

(a) Coarseness

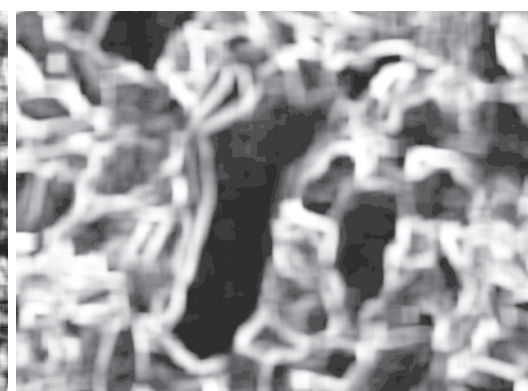

(b) Contrast

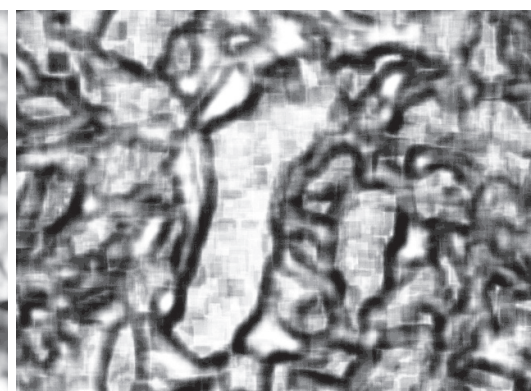

(c) Business

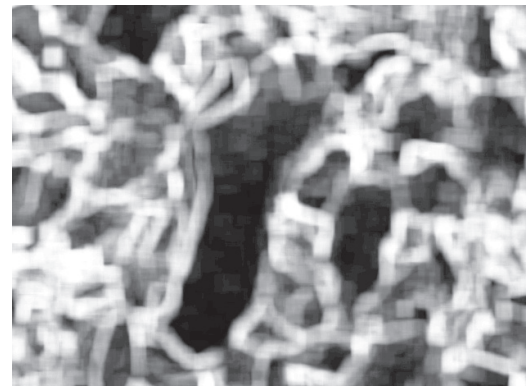

(d) Complexity

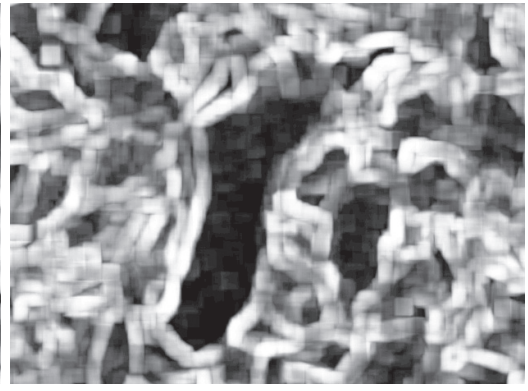

(e) Strength

Fig. 3. GTDM: visualisation of texture feature maps.

feature maps obtained for each feature are presented in Fig. 4. When compared to others visually, they seem less informative due to their low resolution and noise.

\section{Laws' energies}

Laws' energy maps are texture descriptors derived from very local information transformed with small convolution kernels (Laws, 1980). The local windows measure the amount of variation concentrating on some repeatable qualities in the image, e.g. levels, spots, edges, ripples, and waves. These qualities are defined as a 5-element vector, and two of them should be multiplied to obtain a kernel. In the presented work all possible combinations of two kernels (25 features) were considered.

\section{CLASSIFICATION}

Each of the introduced techniques enables the description of a pixel with a vector of features. Therefore, it is a straightforward idea to use this knowledge to build a classification model. Since it is difficult to state which approach from the machine learning domain would give the best performance, it was decided to verify the classification accuracy for k-nearest neighbours, kNN (Murphy, 2013), support vector machines, SVM (Burges, 1998), and artificial feed-forward neural networks, ANN (Gurney, 1997).

The kNN method assumes that a verified feature vector belongs to the class which is represented by the majority of vectors found in the closest neighbourhood. Parameter $k$ defines the number of vectors considered in voting and this should be odd number when the two-class problem is addressed. Since the optimal number of neighbours is task dependent, it is not possible to choose freely. In the presented experiments, the optimal value of this parameter was searched for in the range $[1, \cdots, 25]$. Next, when searching for the nearest neighbouring feature vectors, various metrics could be applied. In the presented experiments the following metrics where verified: city block, Chebychev, correlation, cosine, hamming, Jaccard, Euclidean, Mahalanobis, Minkowski, and spearman.

In the case of SVM, training data is used to calculate a hyperplane which divides the hyperspace into two regions assuring that feature vectors belonging to one class occupy only one region. Additionally, attention is paid to ensure that there is as big a margin as possible around the hyperplane to ensure that new data supplied in testing is found on the proper side of the class-dividing hyperplane. In order to ensure the finding of such a linear hyperplane the data is moved to a higher dimension or when this is not sufficient a kernel trick is applied, which roughly speaking changes the linear kernel function into a radial-basis function, RBF, or any function supplied by the user. The appropriate function depends on the data and should be selected for each experiment. In the presented experiments when the linear kernel was applied, a $c$ parameter influencing the margin should be selected and this was searched for in the range 


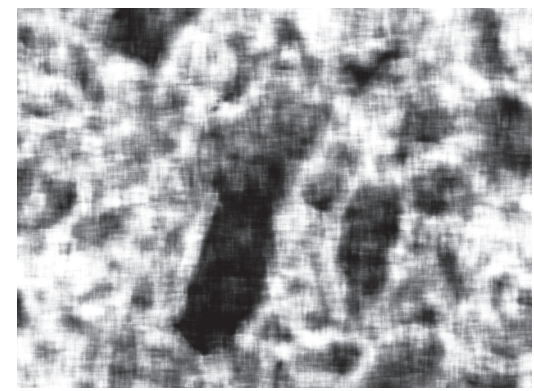

(a) Short run emphasis

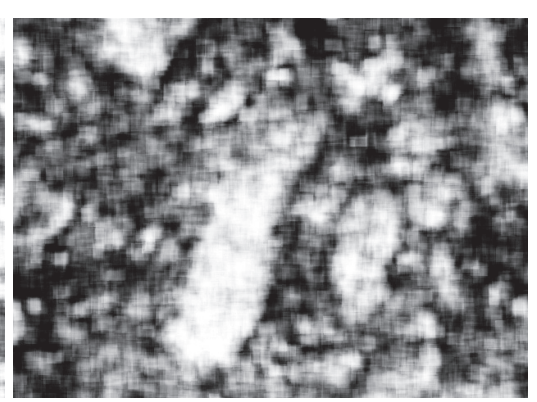

(b) Long run emphasis

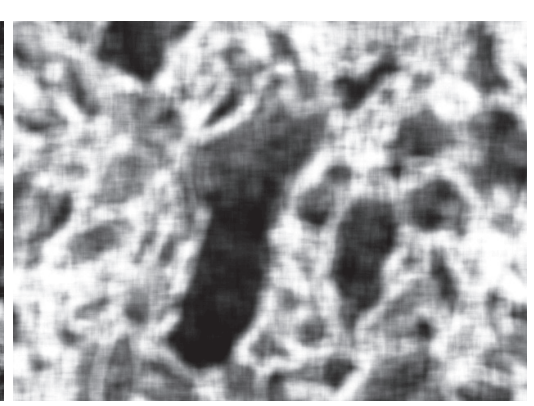

(c) Grey level non-uniformity

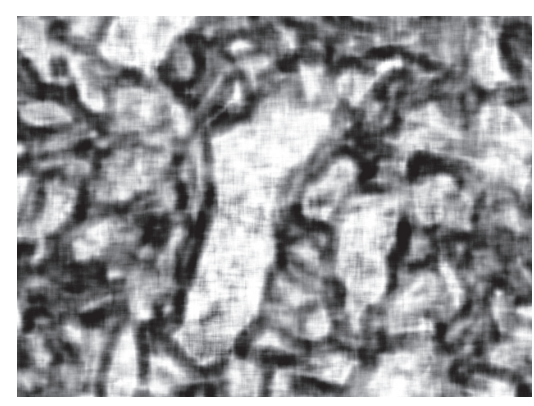

(d) Low-grey level run emphasis

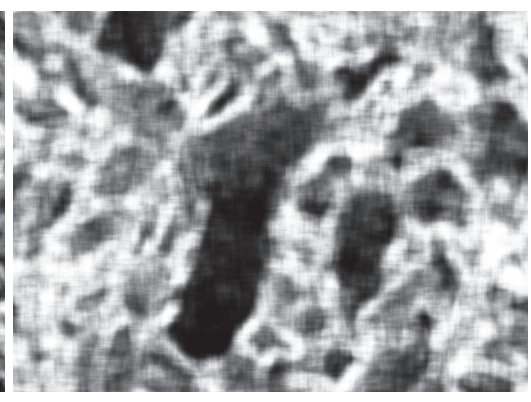

(e) Run percentage

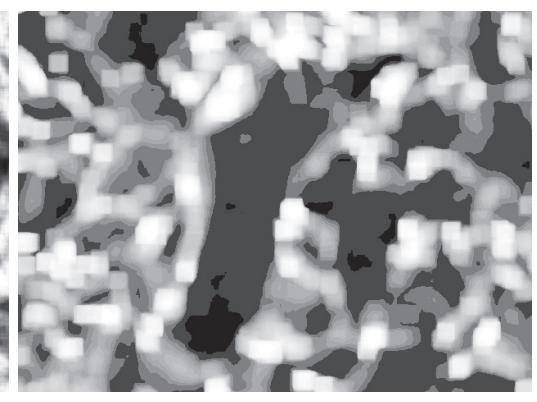

(f) High grey level run emphasis
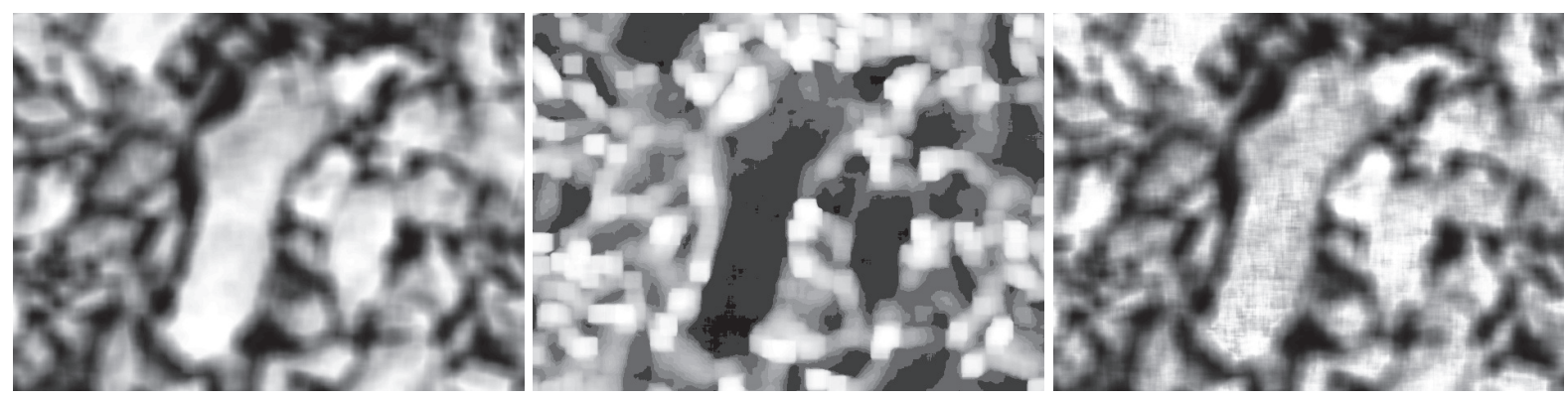

(g) Short run low grey level run emphasis (h) Short run high grey level run (i) Long run low grey level run emphasis emphasis

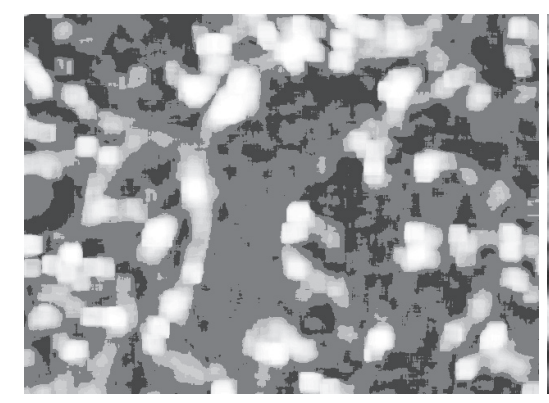

(j) Long run high grey level run emphasis

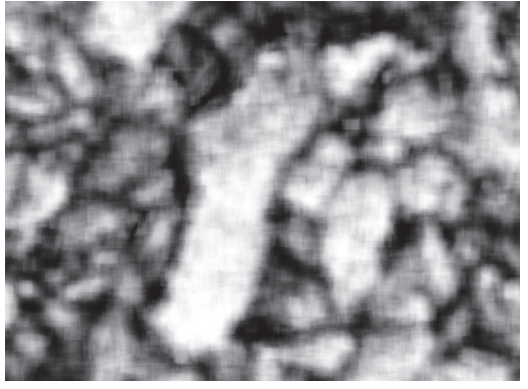

(k) Run length non-uniformity

Fig. 4. RLM: visualisation of texture feature maps. 
$\left[2^{-5}, \cdots, 2^{5}\right]$. When applying RBF except for the $c$ parameter (which was searched for in the same range as the linear kernel) the $\gamma$ parameter, defining the function shape, should also be determined and its value was searched for in the range $\left[2^{-5}, \cdots, 2^{5}\right]$.

Another approach for data classification is presented by ANN method. Here the whole system is constructed with perceptrons/neurons. The perceptron is a mathematical function which calculates an answer on the basis of input data. The high quality reasoning of the network is due to the neuron connections into layers. In the case of fully-connected artificial neural networks, all answers from one layer become the inputs for all the neurons in the second layer. There are weights associated with each input, and their values are optimised in the training process which is realised with a back-propagation method. There are several methods which can be applied in this step, we have decided to use the LevenbergMarquardt back-propagation method. In order to fit this classification to the problem architecture can be changed, this means that the number of layers and the number of perceptrons per layer can be chosen. All the layers which are between the data layer, whose length corresponds to the feature vector, and the output layer, whose number of answers is related to the count of distinguished classes, are named hidden layers. In the presented experiments from 1 to 3 hidden layers were used, and each layer has 5 or 10 neurons.

\section{IMAGE ACQUISITION}

The images used in the research show the rock material gathered from the coal waste dump "Rymer", Poland. The material mainly consisted of gangue (clay slate), which is a typical mineral matter accompanying coal deposits. During sample gathering, special attention was paid to ensure uniformity and representativeness. Image acquisition was performed using a Nikon D80 digital camera. The material was placed in a metal box $(370 \times 320 \times 100 \mathrm{~mm})$ and the surface of the rock material was formed so that it was as flat as possible. Pictures were taken in ambient light conditions. Finally, 32 pictures with $484 \times 648$ pixels resolution were obtained. As the gangue is grey, it was decided that all images should be converted into HSV (hue, saturation, value) colour space and only use the value channel for further processing. Slight differences in illumination were eliminated by filtering the images with a Gaussian filter with a standard deviation of $\sigma=$ 30. The result was then subtracted from the original image. For all these images, manual annotation is supported.

\section{RESULTS}

This research concentrates on several experiments which show the applicability of image texture features for rock body segmentation. Firstly, a comparison is made of the classification performance of feature vectors obtained for each texture operator: first-order features, FOF, second-order features, COM, run-length matrix, RLM, grey-tone difference matrix, GTDM, and Laws' energies, LAWS. In this experiment not only is the data description method evaluated, but the best classification method is also evaluated whilst considering a wide range of parameters. Next, whether it is possible to improve results selecting features manually is evaluated. Data reduction with principal component analysis, PCA, is also exploited to shorten the feature vector and derive the most descriptive information. Finally, the fusion of features is considered. To better understand the results, a visual inspection of the achieved segmentation is given.

All experiments were prepared in the Matlab 2016b environment and run using Windows 10 on a computer with an Intel ${ }^{\circledR}$ i7 processor and $32 \mathrm{~GB}$ of RAM. The implementation of textural features used existing codes to calculate COM features and the authors implemented methods for all the other features. For classification purposes the kNN and ANN methods from the Statistics and Machine Learning Toolbox ${ }^{\mathrm{TM}}$ were applied, while for SVM the libSVM library (Chang and Lin, 2011) was exploited.

In the experiments, the dataset was divided into four equal parts. The data from the first three parts were used for training using three-fold cross-validation regime. The data from the fourth part was used as a validation set. Since the classification is based on features calculated per pixel, a large amount of data was accessible (ca. $3 \cdot 10^{7}$ ). However, this data was highly imbalanced $(10: 1)$, therefore it was decided to randomly sample the class with a larger number of representatives (rock interior) to select the same number of samples that characterised the second class (rock border). The accuracy of classification was calculated as a correct classification ratio which is the ratio between correctly detected borders and interiors to all the considered samples. The results are described by the average accuracy and the standard deviation. The results are given separately for the training and validation sets.

\section{TEXTURE DESCRIPTIVE STRENGTH EVALUATION}

In the presented approach, the image segmentation is a result of the classification of each image pixel 

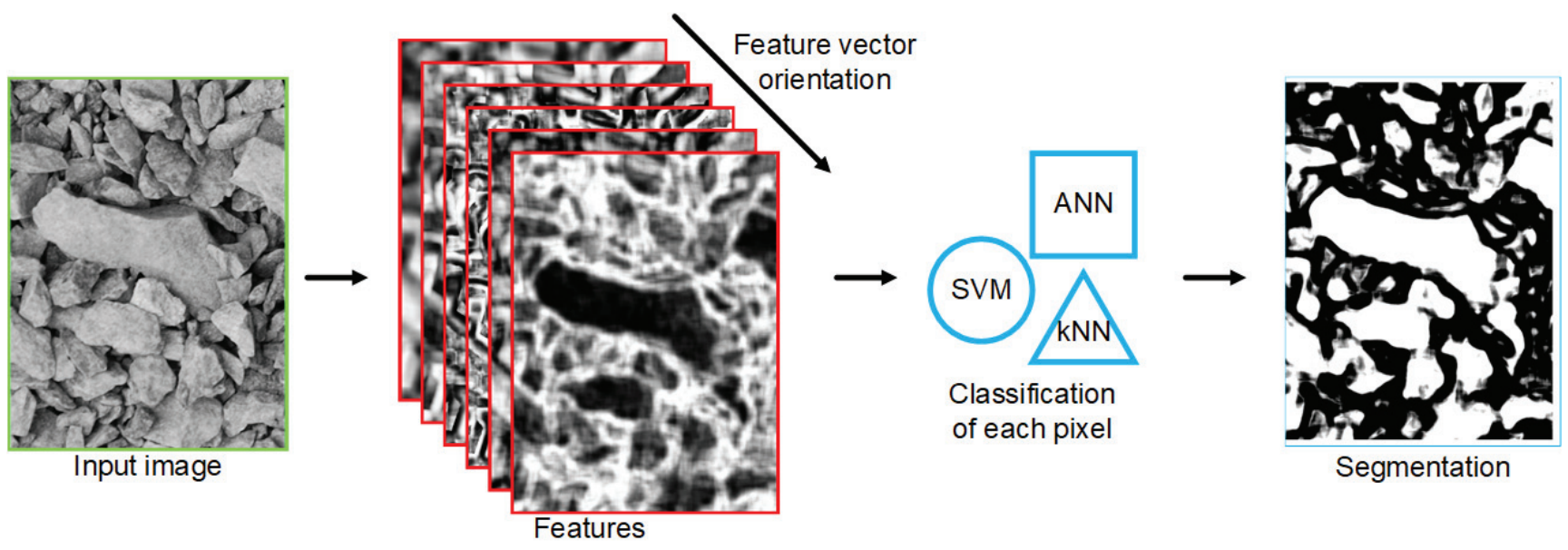

Fig. 5. Data flow in experiments concentrating on separate analysis of textural features.

Table 1. The best result of classification between the rock border and interior for each considered texture description method when the kNN classifier was applied.

\begin{tabular}{lrrlrr}
\hline Method & Feature No & $\mathrm{k}$ & Metric & Acc+Std: Training & Acc+Std: Validation \\
\hline COM & 14 & 25 & Chebychev & $\mathbf{0 . 7 4 5 4} \pm \mathbf{0 . 0 0 6 7}$ & $\mathbf{0 . 7 5 0 0} \pm \mathbf{0 . 0 0 1 1}$ \\
OOM $_{H}$ & 14 & 25 & Chebychev & $0.7367 \pm 0.0064$ & $0.7457 \pm 0.0009$ \\
FOF & 6 & 25 & City block & $0.7410 \pm 0.0057$ & $0.7437 \pm 0.0014$ \\
FOF & 6 & 25 & City block & $0.7293 \pm 0.0067$ & $0.7323 \pm 0.0051$ \\
GTDM & 5 & 25 & City block & $0.7396 \pm 0.0042$ & $0.7402 \pm 0.0011$ \\
GTDM & 5 & 25 & Euclidean/Minkowski & $0.7250 \pm 0.0113$ & $0.7278 \pm 0.0029$ \\
LAWS & 25 & 25 & Chebychev & $0.7471 \pm 0.0054$ & $0.7503 \pm 0.0016$ \\
RLM & 11 & 25 & City block & $0.7348 \pm 0.0067$ & $0.7391 \pm 0.0009$ \\
RLM $_{H}$ & 11 & 25 & City block & $0.7271 \pm 0.0054$ & $0.7379 \pm 0.0011$ \\
\hline
\end{tabular}

into the rock border or rock interior class. Therefore, the accuracy achieved during the classification process also reflects the quality of the segmentation. Firstly, we have evaluated the performance of the considered approaches for texture description and the influence of the classification methodology on the outcome. Tables 1, 2, and 3 present the best classification scores while Fig. 5 depicts the data flow schema.

The features calculated by the mentioned methods have various ranges, while classifiers work better when all features are in the same range. Initially, the normalization scaled the data to occupy a given range. When preparing the texture feature map visualisation, it was observed that the rock borders are more visible when the histogram equalisation follows the range normalisation (histogram stretching in the case of an image). Therefore, for both methods of data preparation $\mathrm{kNN}$ was applied (the subscript $H$ denotes data where only range normalisation was applied). Observing the outcomes of the experiments, one could notice that increasing the value of the $k$ parameter improves the classification accuracy. In the presented experiments, several metrics have been used. They have a small impact on the outcomes as the differences for the same number of voting neighbours are in the third decimal place. The results gathered in Tab. 1 show the best performance for each method. For the training and validation sets, a very similar outcome shows the stability of the results. This conclusion is additionally supported by low values of recorded standard deviations of the results in the training set. LAWS texture descriptors have the highest accuracy of $75.03 \%$ and are closely followed by COM descriptors with $75.00 \%$. Additionally, these good results are also confirmed by the corresponding value of the area under the receiver operating curve (AUC) presented in Fig. 6 for the best outcomes reported for each texture descriptor. In every cases, the additional application of histogram equalisation improves the classification score by 1 point. Therefore, in further experiments, only this data preparation method is used. 
Table 2. The best correct classification ratio for methods based on texture features classified with linear SVM.

\begin{tabular}{lrrrr}
\hline Method & Features No & c & Acc+Std: Training & Acc+Std: Validation \\
\hline COM & 14 & $2^{2}$ & $0.7569 \pm 0.0066$ & $0.7618 \pm 0.0002$ \\
FOF & 6 & $2^{-2}$ & $0.7474 \pm 0.0059$ & $0.7521 \pm 0.0001$ \\
GTDM & 5 & $2^{3}$ & $0.7426 \pm 0.0092$ & $0.7502 \pm 0.0005$ \\
LAWS & 25 & $2^{-3}$ & $0.7604 \pm 0.0018$ & $0.7622 \pm 0.0015$ \\
RLM & 11 & $2^{-1}$ & $0.7444 \pm 0.0061$ & $0.7516 \pm 0.0003$ \\
\hline
\end{tabular}

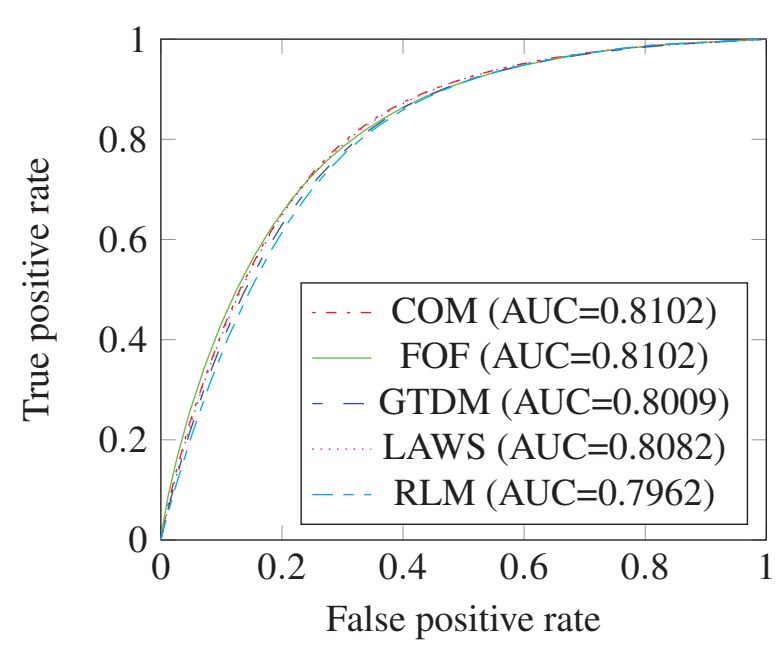

Fig. 6. Receiver operating curve for the best scores recorded for each texture operator with kNN classifier.

Table 2 gathers classification accuracy obtained for the linear SVM approach. Here, the highest correct classification ratio has a level of $76 \%$, and again LAWS features, followed by COM features are better than the others. As in the previous case, the AUC corresponds to the results of Fig. 7. Similarly to the previous case, for each texture descriptor, different settings of the classifier returned the best score. However, none of the compared approaches have shown significantly better outcomes than the others. In the case of RBF-SVM only worse results were achieved, therefore they are not presented.

The performance for ANN classifier is given in Tab. 3 while the receiver operating curve is depicted in Fig. 8. For all texture descriptors except for LAWS, two hidden layers with ten elements gave superior results. It is interesting to observe, that the texture descriptor with the longest feature vector of 25 elements needed only one hidden layer, while the methods described by five or six features achieved better performance when two hidden layers were constructed. Moreover, the smallest network exploited to work with LAWS features gave the best accuracy (77\%) of classification. Yet again, COM performance is very similar.

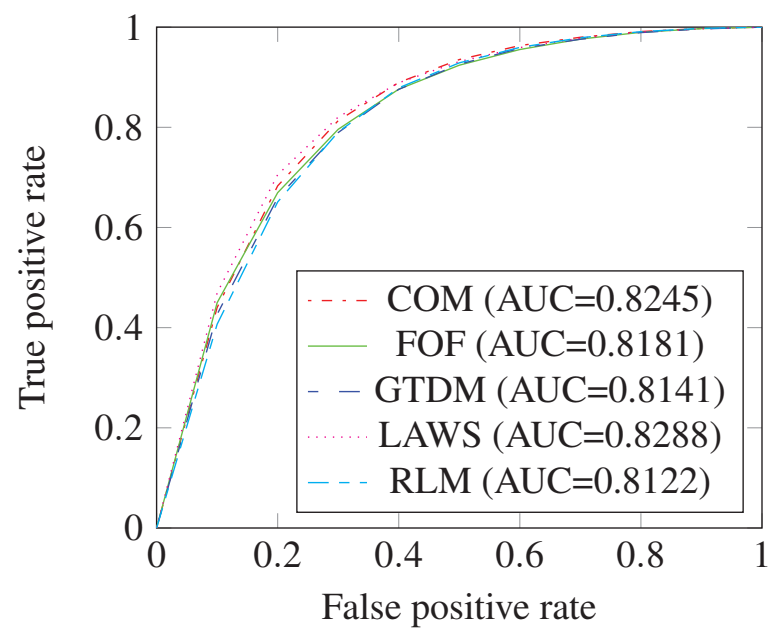

Fig. 7. Receiver operating curve for the best scores recorded for each texture operator with SVM classifier.

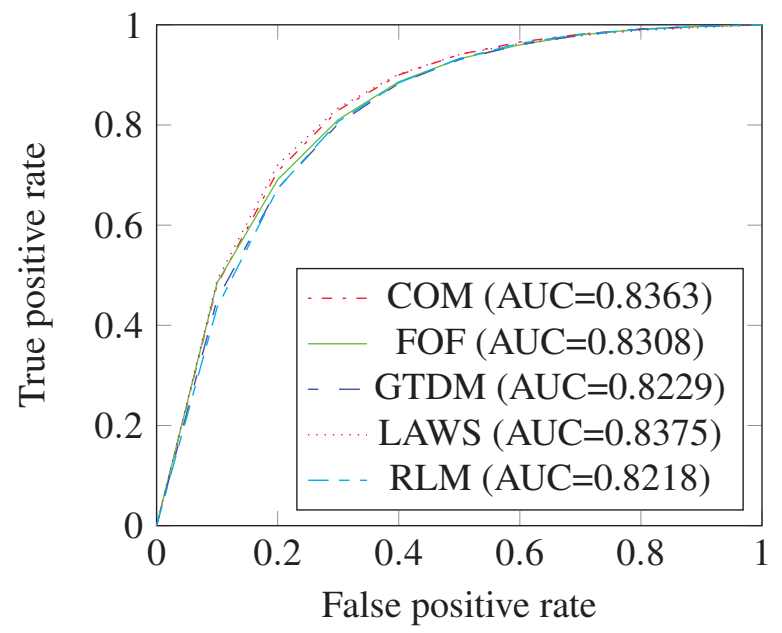

Fig. 8. Receiver operating curve for the best scores recorded for each texture operator with ANN classifier.

When one looks through the features, the similarity between the presented texture feature maps (see Figs. 1-4) can be observed. It is also visible that the borders are much pronounced on some texture feature maps. The visual similarity should correspond to a similar feature description, also when the outline is clearly visible in the data it should be easier for the classifier to detect it. Thus having these facts in mind, a manual selection of distinctive features 
Table 3. The best results of classification recorded for each texture description method when ANN classifier was used. Three evaluation approaches are compared here including: all features generated with the methods, manually selected features, and PCA application.

\begin{tabular}{|c|c|c|c|c|c|c|}
\hline \multirow{2}{*}{ Method } & \multirow{2}{*}{$\begin{array}{c}\text { Hidden } \\
\text { layers }\end{array}$} & \multirow[t]{2}{*}{ Neurons } & \multirow[t]{2}{*}{ Features } & \multirow[t]{2}{*}{ Dimensions } & \multicolumn{2}{|c|}{ Acc + Std } \\
\hline & & & & & Training & Validation \\
\hline \multirow{3}{*}{$\mathrm{COM}$} & \multirow{3}{*}{2} & \multirow{3}{*}{10} & All & 14 & $0.7668 \pm 0.0069$ & $0.7695 \pm 0.0009$ \\
\hline & & & Selected & 10 & $0.7496 \pm 0.0094$ & $0.7575 \pm 0.0006$ \\
\hline & & & PCA & 5 & $0.7569 \pm 0.0058$ & $0.7606 \pm 0.0005$ \\
\hline \multirow{3}{*}{ FOF } & \multirow{3}{*}{2} & \multirow{3}{*}{10} & All & 6 & $0.7570 \pm 0.0070$ & $0.7580 \pm 0.0013$ \\
\hline & & & Selected & 5 & $0.7492 \pm 0.0079$ & $0.7540 \pm 0.0006$ \\
\hline & & & PCA & 5 & $0.7562 \pm 0.0065$ & $0.7576 \pm 0.0011$ \\
\hline \multirow{3}{*}{ GTDM } & \multirow{3}{*}{2} & \multirow{3}{*}{10} & All & 5 & $0.7528 \pm 0.0062$ & $0.7546 \pm 0.0012$ \\
\hline & & & Selected & 4 & $0.7423 \pm 0.0047$ & $0.7448 \pm 0.0014$ \\
\hline & & & PCA & 4 & $0.7492 \pm 0.0056$ & $0.7526 \pm 0.0018$ \\
\hline \multirow{3}{*}{ LAWS } & \multirow{3}{*}{1} & \multirow{3}{*}{10} & All & 25 & $0.7666 \pm 0.0053$ & $0.7707 \pm 0.0004$ \\
\hline & & & Selected & 10 & $0.7401 \pm 0.0142$ & $0.7459 \pm 0.0024$ \\
\hline & & & PCA & 8 & $0.7664 \pm 0.0035$ & $0.7674 \pm 0.0043$ \\
\hline \multirow{3}{*}{ RLM } & \multirow{3}{*}{2} & \multirow{3}{*}{10} & All & 11 & $0.7544 \pm 0.0061$ & $0.7576 \pm 0.0002$ \\
\hline & & & Selected & 6 & $0.7522 \pm 0.0060$ & $0.7552 \pm 0.0002$ \\
\hline & & & PCA & 7 & $0.7523 \pm 0.0064$ & $0.7561 \pm 0.0006$ \\
\hline
\end{tabular}

was performed: for the FOF texture operator the mean was removed as its border presentation was the weakest one; for COM the variance, sum average, sum variance, and information measure of correlation I were neglected; for GTDM the coarseness seems to convey less valuable information; for LAWS the number of applied masks was reduced to 10 out of the initial 25; for RLM the short run emphasis, long run emphasis, short-run low grey level run emphasis, short-run high grey level run emphasis, and long run high grey level run emphasis were omitted. Then the classification performance for those selected features for the best from previously presented classification methods (ANN) was evaluated and the results are presented in the rows named Selected in Tab. 3. The manual selection of valid parameters proved to worsen the correct classification ratio. Yet it was only based on the visual inspection of the texture feature maps. On the other hand, the dimension reduction can be obtained by application of PCA which aims to find those orthogonal directions, where the data is characterised by high variability. Choosing directions of the highest variability and simultaneously removing those modes of low variation enables the reduction of the data dimensionality whilst keeping a similar amount of information. In the presented research PCA was applied before ANN classifier. It assumed the use of those directions which constitute at least 99\% of the information included in the original data. The outcomes achieved for this approach are gathered in Tab. 3 in rows annotated as PCA. Here again, a deterioration of classification performance is noticeable when compared to the results obtained for all features. Feature dimension reduction with PCA also did not improve the results. However, it noted better performance when compared to manually selected features. In the case of LAWS and COM, PCA reduced the number of modes achieving higher accuracy.

The distinction between the rock border and interior, when described by various texture operators seems to have similar accuracy with the exception of the applied classifier. Moreover, the best performing methods depend on the applied classification technique. However, LAWS and COM texture operators show very similar performance, and with such small discrepancies, it is difficult to decide which is better.

\section{MULTI-TEXTURE APPROACH}

The description of image content derived by the texture operator varies between the techniques considered in this work. The experiments presented in the previous section, showed that it is possible to distinguish between the rock interior and border, however, a considerable amount of false positives is noticeable. Yet, a major number of false positives are located near the borders, making the border line thicker than in manual annotation. While the minority reflects very local edges on the rock surface. Both of those cases do not constitute a problem, as 


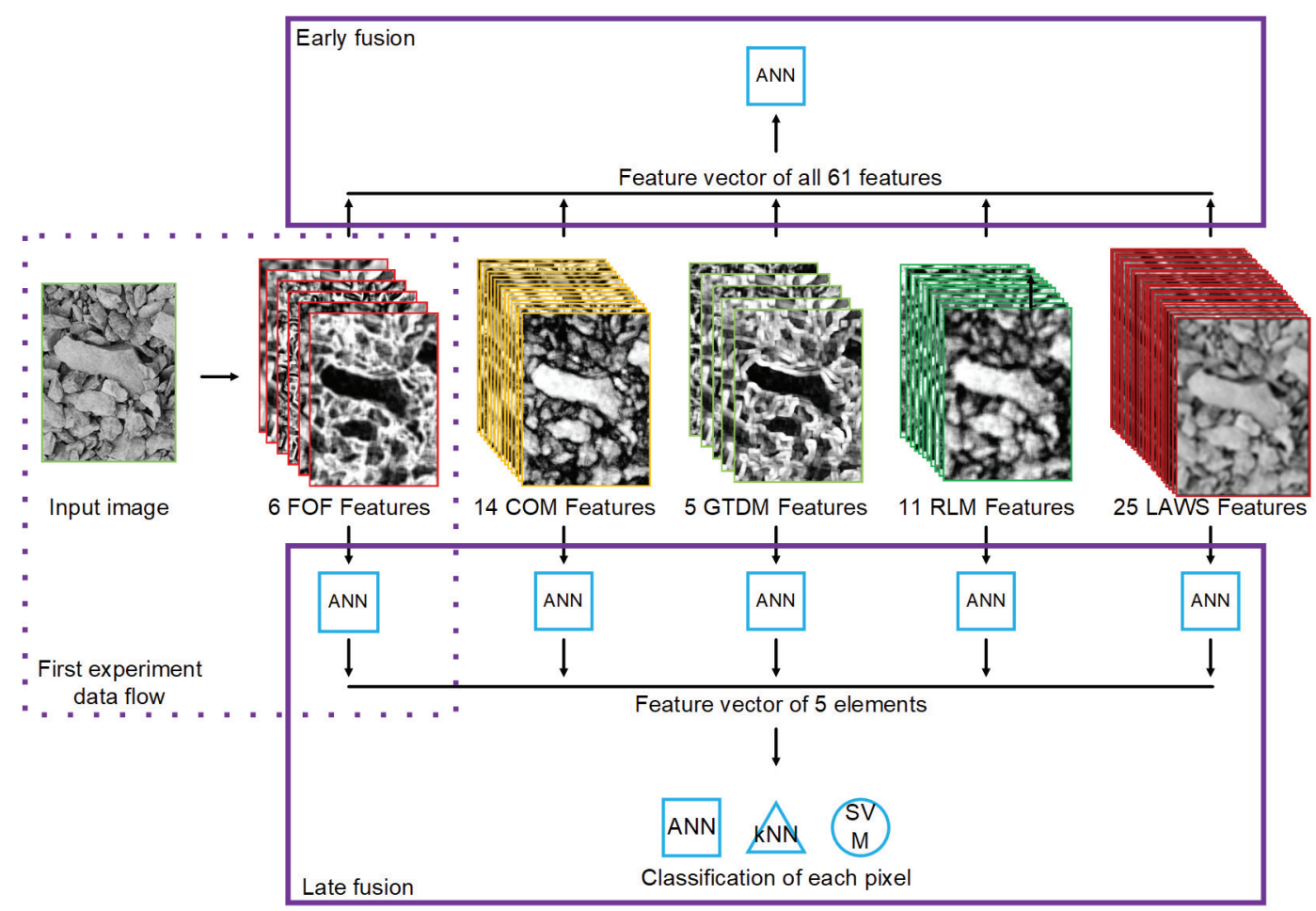

Fig. 9. Data flow in early- and late-fusion.

Table 4. The table gathers results recorded in the experiments with a multi-texture approach. It compares the performance of early-and late-fusion feature analysis and the influence of feature reduction with PCA (brackets enclose the number of selected features).

\begin{tabular}{cccrr}
\hline Approach & Classifier & Parameters & \multicolumn{2}{c}{ Acc+Std } \\
& & & Training & Validation \\
\hline Early-fusion & ANN & 1 layer, 10 neurons & $0.7901 \pm 0.0094$ & $0.7919 \pm 0.0051$ \\
\hline \multirow{2}{*}{ Late-fusion } & ANN & 2 layers, 10 neurons & $0.7727 \pm 0.0046$ & $0.7742 \pm 0.0004$ \\
& kNN & 25, any metric & $0.7324 \pm 0.0089$ & $0.7379 \pm 0.0048$ \\
& SVM & $2^{-2}$ & $0.7604 \pm 0.0018$ & $0.7611 \pm 0.0000$ \\
\hline
\end{tabular}

they could be easily removed if necessary in postprocessing using simple skeletonisation or a best-fit (Piorkowski, 2017) approach. Yet, in order to limit the importance of post-processing, the number of false positives should be diminished. Therefore, a classifier based on a combination of all the presented texture operators approaches is presented. In this experiment, two methods for feature combination are taken into account: early- and late-fusion. In the case of the early-fusion approach, 61 features derived for texture analysis approach presented in the previous experiment are considered all together and constitute one feature vector fed for a classifier as depicted at the top of Fig. 9. For late-fusion, a cascade of classifiers is applied. In the first stage the best classifier for each texture operator described in the previous section is applied (see details in Tab. 3) and then the responses (probabilities to belonging to one of the two classes) constitute a feature vector fed to the second stage as presented in the bottom of Fig. 9.

Table 4 gathers the outcomes obtained for the multitexture approach. The best performance was recorded for the early-fusion approach and reached $79 \%$, this score is also achieved by the AUC presented in Fig. 10. In this approach, other classifiers were not considered, since their weaker performance in an individual texture operator would be repeated in this case too. On the other hand, when the late-fusion method was evaluated, a slight improvement over individual approach is visible and reaches from $73 \%$ for $\mathrm{kNN}$ classifier to $77 \%$ for ANN. 


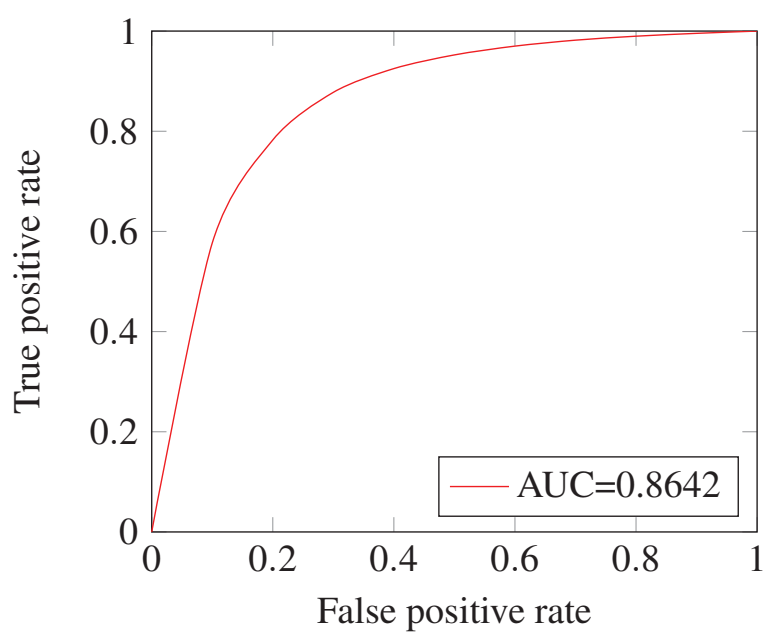

Fig. 10. Receiver operating curve for the best score Early fusion with ANN.

It is interesting to observe, that ANN classifier using all features to build the model gives the best outcome, significantly improving the accuracy. While some feature maps when analysed visually seemed similar, in reality, there were some slight differences, that could be used by ANN to achieve better performance. This finding is also supported by the worse performance of classifiers trained with a reduced number of features.

\section{VISUAL INSPECTION}

Visual inspection is another approach to check segmentation accuracy using the best approach presented in the previous section. Fig. 11 presents some examples, where the original image is the background for inpainted contours obtained from manually prepared masks and those resulting from image segmentation. The red colour denotes pixels which were classified as borders, while blue corresponds to borders drawn manually. When the manual segmentation overlaps with the automatic one a white colour is applied.

It can be observed, that the segmentation is satisfactory and the automatic segmentation (red colour) overlaps the manual annotation (blue colour) in most regions of the examples shown. The proposed segmentation very well outlines the bodies of large stones, however, the small ones are recognised as borders. This is the result of applying texture features as a description method. Because in order to compute a feature, a region of $21 \times 21$ pixel resolution is considered, therefore one cannot assume that the automatic segmentation will not suffer from such a feature derivation approach. There is also another problem which can be seen in the visualisation. There are regions where the border was detected in the middle of the rock body. This is due to a small collapses in the rock surface, which generate local borders. Sometimes, when stones of a similar colour are placed near to each other, such a border may not be recognised correctly too.

Though the presented results do not solve the segmentation problem, they do show that most of the large rocks borders have been properly identified. It has to be noted, that the distinction between individual rocks, with similar colour properties is sometimes difficult even for an expert. While the determination of sharp contours seems to not be possible using the proposed textural approach, the obtained results can have a practical application. First of all, the method enables for the determination of large grain content in the total material. Such information is useful from a practical point of view. In many technological processes in the raw material industry, the biggest grains have to be ground. A priori assessment of the amount of material that is supposed to be ground enables the assessment of associated costs and machinery wear. Secondly, the presented images show that the segmentation obtained forms a good basis for grain sizes determination. Finally, the results justify the application of texture methods for rock grain segmentation, despite the aforementioned limitation of its resolution.

\section{DISCUSSION}

There is not an abundance of research that considers rock material segmentation in the literature. The computer scientists focus on the usage of textural features for the determination of rock type (Lobos et al., 2016; Perez et al., 2011; Tessier et al., 2007). Perez et al. (2011) is a good representation of this approach and is a further development of the idea presented by Tessier et al. (2007). The rock grains depicted in the images are segmented using morphological operations along with a watershed algorithm. The textural features, along with the colour ones, are used for discriminating the rocks having different grind-ability. The determination of rock size grade is less common. The typical approach is based on a material sieved through a set of screens. The screen mesh sizes get progressively smaller. The screens allow only material of particle sizes smaller than their mesh size to pass through. Therefore, the material is divided into a set of fractions of granularity determined by consecutive mesh sizes. This procedure is usually used in the raw material processing industry for particle size distribution determination. Each fraction is photographed and 

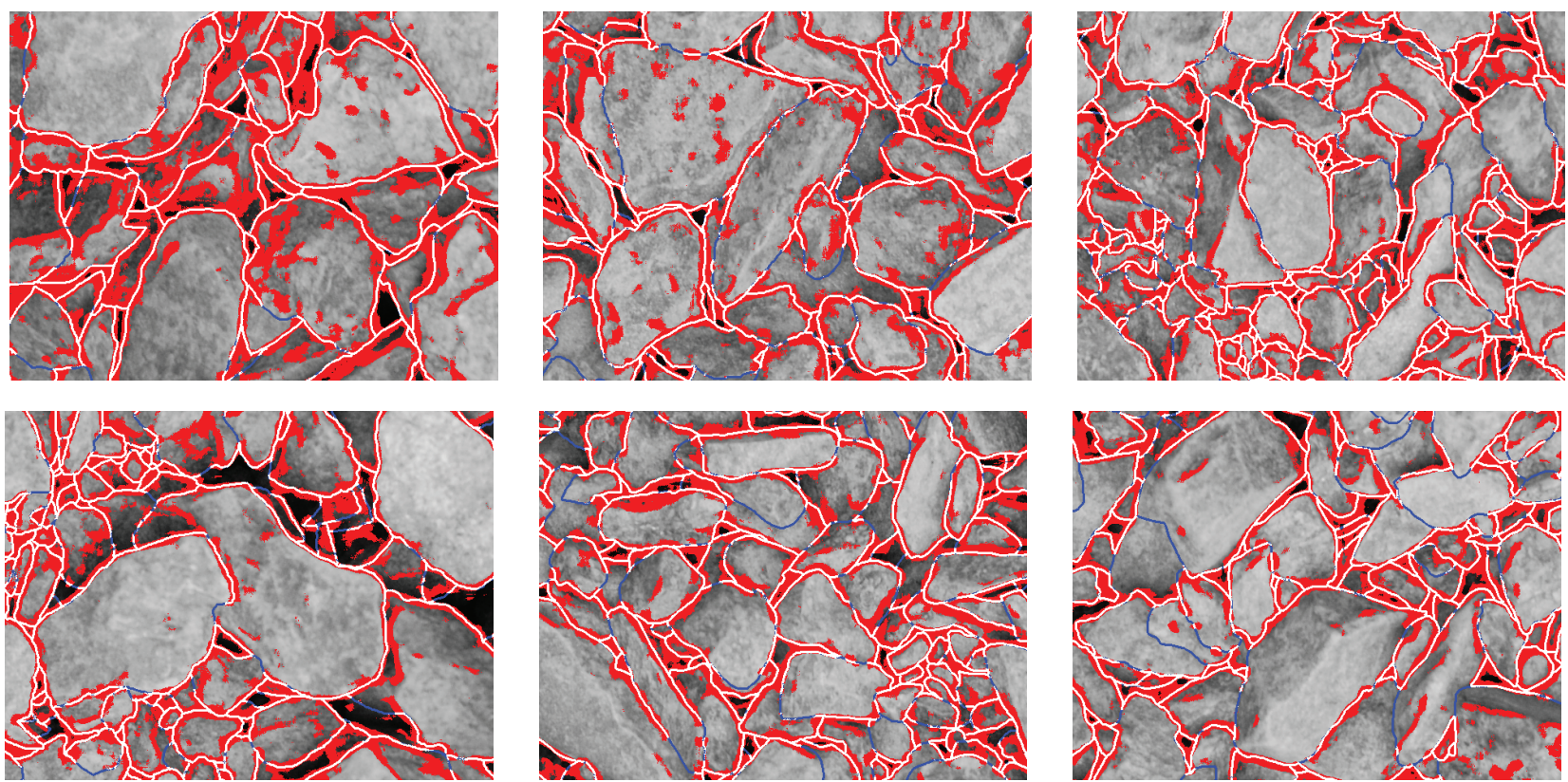

Fig. 11. Visualisation of segmentation results in six different images. The red colour shows pixels classified as rock border. The blue colour depicts manual annotations. The white colour indicates overlap between automatic segmentation and manual segmentation.

used as an input for features extraction and machine learning algorithms. Some authors define as much as 12 different fractions (Murtagh et al., 2005), while others use just 2 or 3 (Aldrich et al., 2010; Kistner et al., 2013). The comprehensive list of pieces of work addressing this topic was presented by Yaghoobi et al. (2019). The approach though interesting and obtaining moderately good results, appears to be difficult in practical application. The run-of-mine, ROM, usually contains the whole spectrum of grades mixed together. A ROM composed of a narrow fraction of grain sizes is virtually impossible. Therefore, an approach neglecting the grain boundary seems to be applicable only in selected, special cases. On the other hand, the determination of grain boundary is universal. Although, the application of texture based methods is barely present in the literature.

Comparison between texture and other methods of determination of grain boundary shows that the latter performs better in the case of small grain border detection, while frequently erroneously detecting boundaries inside the bigger rock grains. The presented work showed that in most cases the texture operator method is resistant to that situation. However, it tends to neglect fine grains, which could be improved with a higher resolution of input data. This problem results from the resolution of the window which is used to calculate the texture features. Because at least $21 \times 21$ pixel resolution is necessary, that makes it difficult to discriminate rocks that are smaller that this size.
It must be mentioned, that many authors present results visually (e.g. (Wang, 2008; Thurley, 2013)), causing the comparison to be less precise. Nevertheless, it seems that the proposed method is slightly better at precise grain borders delineation than the others presented in the literature.

Having said that, the one exception has to be pointed out. (Outal, 2006) presented comprehensive research analysing various approaches using the morphological transformations (e.g. computation of geodesic maps). The best solution uses the modified ultimate opening transformation. The transformation is modified in that sense, that the associated function (called granulometric function) is constructed to recover the critical disk (Outal and Beucher, 2009) contained in each rock fragment. The disks are computed for successive structural element size $k$. The parameter also corresponds to the value of granulometric function. In conclusion, the author proposes the use of local maximum values of granulometric function as the markers for the watershed algorithm. The presented applications on real rocks images show the potential of the method. However, it has to be noticed, the process of constructing the markers is not simple. In spite of the usage of only a limited set of morphological operators (erosion, dilation, ultimate opening, etc.) the algorithm is sophisticated and the appropriate choice of two parameters is necessary. The maximum size of the grain should also be known (the stop condition for ultimate opening computation). The presented 
algorithm performs reasonably well in most situations. However, there is a risk of identifying the internal structure of a bigger rock texture being the finest grain class. The approach also may have problems with closely placed rock fragments of very similar surface textures (e.g. two flat fragments of the same rock placed one on another). Therefore, the texturebased methods should be at least used as a support for the morphological procedure. The big advantage of the algorithm is its resistance to the edges appearing inside the rock grains, which are not their edges. This approach can successfully characterise the bulk material, assessing, for example, a number of fine grains.

An important topic that must also be addressed is the issue of correspondence between the results of determining the grain size based on the registration of the top layer of rock material and the values obtained as a result of sieve analysis. As it was presented in Outal et al. (2008), the obtained grain surface distributions, presented in the form of a histogram, differ from the analogous histograms regarding volume obtained from the sieve analysis. However, a function transforming the areas distribution in the volume distributions can be elaborated. Outal et al. (2008) proposed using a linear relationship to calculate the values determined by the size of the image and the sizes determined based on the sieve analysis. The grain size, determined based on the image, is represented by a disk that can be entered in the outlined grain. In the case of sieve analysis, this corresponds to a grain that can pass through a sieve with a specified mesh size. Analysis of the results of comparing the results of the sieve analysis and the use of image-based size histogram mapping indicates that satisfactory results can be obtained if the histograms do not divide the size range into too many ranges. The greater the number of histogram intervals, the greater deviations can be observed. It should be noted, however, that in practice only the coarse division into such compartments is used. For such a case, the use of the mapping proposed in Outal et al. (2008) seems sufficient.

The knowledge of the sizes of raw material grains is utilised in many different ways during excavation and processing. The applicability of the presented results differs depending on which part of processing is considered. The proposed method seems to be well suited to the determination of big rock grains among finer bulk material. This is useful during a few stages of raw material production. First of all, the big grains often require crushing immediately after excavation (e.g. by blasting). Normalising upper grain size at the beginning of the process is important for effective transportation and further enhancement.
Secondly, the rock material is often separated by screening. As the parts of sieving screens become worn out, oversized particles can remain in sieved material. This can be detected by the presented approach. Finally, the method can be used as a part of quality monitoring for processes, where uniform or at least upper limited granularity is essential. Aggregate production is an example of this. For each presented potential application, the achieved accuracy of the method is sufficient. However, the method has to be developed further to be applicable for fine grain content determination or application for the precise determination of particle size distribution.

The presented work shows that, in general, the approach can lead to interesting results. The achieved accuracy is good enough for practical applications in selected areas. However, the problem of grain size determination using texture features is far from being solved and the method requires improvement.

\section{CONCLUSIONS}

The work describes research addressing the problem of the automatic segmentation of grains which applies the image analysis approach. The proposed method derives descriptive information calculating the texture features for images representing grains. In contrast to the standard application of texture operators, the feature values are computed for each image pixel and not for the whole image. The experiments evaluate the descriptive properties of first-order features, second-order features, grey-tone difference matrix, run-length matrix, and Laws' energies and do not mention local binary patterns and local phase quantisation methods which proved to be too weak for this comparison. The segmentation is achieved by the classification of each pixel to one of two considered classes: grain border and interior. Additionally, the experiments tried to answer the question which from machine learning methods would be the most beneficial for this purposes. Therefore, the k-nearest neighbour, support vector machine, and artificial neural networks were evaluated as classifiers.

The segmentation evaluation was realised in three steps. Firstly, feature vectors derived using a single texture operator were evaluated with the application of all the mentioned classifiers. This enabled information about which classifier is the most beneficial and what its optimal hyperparameter settings are to be obtained. All the experiments performed revealed that ANN classifier is most promising and reaches more than $75 \%$ correct classification ratio for all the mentioned methods, while achieving around $77 \%$ 
for COM and LAWS methods. Secondly, whether combining information derived from all texture operators can improve the classification accuracy and as a consequence make the segmentation more precise was tested. Two solutions for data flow were considered: early- and late-fusion. Here both approaches improved the classification accuracy, but the best results were obtained for the early-fusion of $79 \%$. Additionally, the authors verified in the experiments of the first and second step, that manual feature selection or data dimension reduction with PCA is inapplicable. Finally, visual inspection proved that the bodies of grains are nicely segmented, while the borders are rather thick and ragged. Yet, the borders follow manual annotations and thus represent satisfactory segmentation.

One of the most significant problems encountered during the research was the lack of a sufficiently large, high-quality database of rock grain images. Therefore, planned future work will include the preparation of such a database at the very first stage. Though it is not strictly a scientific activity, a well-established ground truth is crucial for future scientific work. The presented research shows, that it is possible to obtain good grain segmentation, yet there is still some room for improvement. This can be achieved in two ways. On one hand, the authors plan in further research (when a larger data set is available) to address semantic segmentation as a method of segmentation. On the other hand, methods improving current results in the post-processing stage should be addressed, which concentrate on border thinning and the automatic closing of the delineated contours. There is also research planned in which textural and colour features will be jointly tested for rocks border delineation.

\section{CONTRIBUTIONS}

$\mathrm{KN}$ prepared and executed all of the experiments, analysed the results and prepared images. SI prepared the database and analysed the results. Both authors prepared the manuscript.

\section{ACKNOWLEDGEMENT}

KN research was supported by the Quality Grant of the President of Silesian University of Technology (02/020/RGJ19/0168) and this work was co-financed by SUT grant for maintaining and developing research potential. SI research was supported by the statutory activity of the Central Mining Institute: Application of image recognition and machine learning methods for Run-Of-Mine and waste mineral matter characterisation - No. GIG: 11010117 144.

\section{CONFLICT OF INTERESTS}

The authors declare no conflict of interest.

\section{ABBREVIATIONS}

The following abbreviations are used in this manuscript:

$\begin{array}{ll}\text { ANN } & \text { artificial neural network } \\ \text { AUC } & \text { area under ROC } \\ \text { COM } & \text { second-order features } \\ \text { FOF } & \text { first order features } \\ \text { GTDM } & \text { grey tone difference matrix } \\ \text { HSV } & \text { hue, saturation, value (colour space) } \\ \text { kNN } & \text { k-nearest neighbour } \\ \text { LAWS } & \text { Laws' energies } \\ \text { PCA } & \text { principal component analysis } \\ \text { RLM } & \text { run length matrix } \\ \text { ROC } & \text { receiver operating curve } \\ \text { ROM } & \text { run of mine } \\ \text { SVM } & \text { support vector machine }\end{array}$

\section{REFERENCES}

Ailleres L, Champenois M, Macaudiere J, Bertrand J (1995). Use of image analysis in the measurement of finite strain by the normalized Fry Method: geological implications for the 'Zone Houillère' (Briançonnais zone, French Alps). Mineral Mag 59:179-87.

Al-Thyabat S, Miles N (2006). An improved estimation of size distribution from particle profile measurements. Powder Technol 166:152 - 160.

Aldrich C, Jemwa G, Van Dyk J, Keyser M, Van Heerden J (2010). Online analysis of coal on a conveyor belt by use of machine vision and kernel methods. Int J Coal Prep Util 30:331-48.

Alpana, Mohapatra S (2015). Automated coal characterization using computational intelligence and image analysis techniques. In: 2015 Communication, Control and Intelligent Systems (CCIS).

Alpana, Mohapatra S (2016). Machine learning approach for automated coal characterization using scanned electron microscopic images. Comput Ind $75: 35-45$. 
Amadasun M, King R (1989). Textural features corresponding to textural properties. IEEE T Syst Man Cyb 19:1264-74.

Burges CJC (1998). A tutorial on support vector machines for pattern recognition. Data Min Knowl Discov 2:121-67.

Chang CC, Lin CJ (2011). Libsvm: A library for support vector machines. ACM T Intel Syst Tec 2:27:1-27:27.

Chatterjee S, Bhattacherjee A (2011). Genetic algorithms for feature selection of image analysisbased quality monitoring model: An application to an iron mine. Eng Appl Artif Intel 24:786 - 795.

Cord A, Bach F, Jeulin D (2010). Texture Classification By Statistical Learning From Morphological Image Processing: Application To Metallic Surfaces. J Microsc Oxford 239:159-66.

Galloway MM (1975). Texture analysis using gray level run lengths. Comput Vision Graph 4:172 179.

Gurney K (1997). An Introduction to Neural Networks. Bristol, PA, USA: Taylor \& Francis, Inc.

Guyot O, Monredon T, LaRosa D, Broussaud A (2004). Visiorock, an integrated vision technology for advanced control of comminution circuits. Miner Eng 17:1227 - 1235. Communition '04.

Haines TJ, Neilson JE, Healy D, Michie EA, Aplin AC (2015). The impact of carbonate texture on the quantification of total porosity by image analysis. Comput Geosci 85:112 - 125.

Haralick RM, Shanmugam K, Dinstein I (1973). Textural features for image classification. IEEE T Syst Man Cyb SMC-3:610-21.

Hulthén E, Evertsson CM (2009). Algorithm for dynamic cone crusher control. Miner Eng 22:296 $-303$.

Igathinathane C, Ulusoy U (2016). Machine vision methods based particle size distribution of balland gyro-milled lignite and hard coal. Powder Technol 297:71 - 80.

Igathinathane C, Ulusoy U, Pordesimo L (2012). Comparison of particle size distribution of celestite mineral by machine vision volume approach and mechanical sieving. Powder Technol 215-216:137 $-146$.

Kemeny J, Mofya E, Kaunda R, Lever P (2002). Improvements in blast fragmentation models using digital image processing. Fragblast 6:311-20.

Kistner M, Jemwa GT, Aldrich C (2013). Monitoring of mineral processing systems by using textural image analysis. Miner Eng 52:169-77.
Ko YD, Shang H (2011). A neural network-based soft sensor for particle size distribution using image analysis. Powder Technol 212:359 - 366.

Kwan A, Mora C, Chan H (1999). Particle shape analysis of coarse aggregate using digital image processing. Cement Concrete Res 29:1403 - 1410.

Latała Z, Wojnar L (2001). Computer-aided versus manual grain size assessment in a single phase material. Mater Charact 46:227 - 233. STERMAT 2000: Stereology and Image Analysis in Materials Science.

Laws KI (1980). Rapid texture identification. In: Proc.SPIE, vol. 0238.

Lobos R, Silva JF, Ortiz JM, Díaz G, Egaña A (2016). Analysis and Classification of Natural Rock Textures based on New Transform-based Features. Math Geosci 48:835-70.

Lu B, Cui M, liu Q, Wang Y (2009). Automated grain boundary detection using the level set method. Comput Geosci 35:267 - 275.

Lumbreras F, Serrat J (1996). Segmentation of petrographical images of marbles. Comput Geosci 22:547 - 558.

Maerz N (1998). Aggregate sizing and shape determination using digital image processing. In: Center For Aggregates Research (ICAR) Sixth Annual Symposium Proceedings.

Materka A, Strzelecki M (1998). Texture analysis methods - a review. Tech. rep., Institute of Electronics, Technical University of Lodz.

Mertens G, Elsen J (2006). Use of computer assisted image analysis for the determination of the grain-size distribution of sands used in mortars. Cement Concrete Res 36:1453 - 1459. 10th EUROSEMINAR on microscopy applied to building materials, University of Paisley, June 21$25,2005$.

Mlynarczuk M, Ladniak M, Piorkowski A (2014). Application of database technology to analysis of rock structure images. PHYSICOCHEM PROBL MI Vol. 50, iss. 2:563-73.

Mora C, Kwan A, Chan H (1998). Particle size distribution analysis of coarse aggregate using digital image processing. Cement Concrete Res 28:921 - 932.

Murphy KP (2013). Machine learning : a probabilistic perspective. Cambridge, Mass. [u.a.]: MIT Press.

Murtagh F, Qiao X, Crookes D, Walsh P, Basheer PAM, Long A, Starck JL (2005). A machine vision approach to the grading of crushed aggregate. Mach Vision Appl 16:229-35. 
Obuchowicz R, Nurzynska K, Obuchowicz B, Urbanik A, Piórkowski A (2018). Caries detection enhancement using texture feature maps of intraoral radiographs. Oral Radiol .

Ojala T, Pietikainen M, Maenpaa T (2002). Multiresolution gray-scale and rotation invariant texture classification with local binary patterns. IEEE T Pattern Anal 24:971-87.

Outal S (2006). Quantification by image analysis of the size distribution of fragmented rocks: improvment of the morphological extraction of surfaces, improvment of the stereological rebuilding. Thèse, Ecole Nationale Supérieure des Mines de Paris.

Outal S, Beucher S (2009). Controlling the ultimate opening residues for a robust delineation of fragmetned rocks. In: The 10th European Congress of Stereology and Image Analysis, Milan, Italy. 6.

Outal S, Jeulin D, Schleifer J (2008). A new method for estimating the $3 \mathrm{D}$ size-distributioncurve of fragmented rocks out of 2D images. Image Analysis Stereology 27:97-105.

Outal S, Schleifer J, Pirard E (2009). Evaluating a calibration method for the estimation of fragmented rock 3d-size-distribution out of 2d images. In: FRAGBLAST 9-Proc. 9th International Symposium on Rock Fragmentation by Blasting, Granada, 13-17 September.

Perez CA, Estévez PA, Vera PA, Castillo LE, Aravena CM, Schulz DA, Medina LE (2011). Ore grade estimation by feature selection and voting using boundary detection in digital image analysis. Int J Miner Process 101:28 - 36.

Piorkowski A (2017). Best-fit segmentation created using flood-based iterative thinning. In: Choras
R, ed., Image Processing and Communications Challenges 8. IP\&C 2016, vol. 525 of AISC. Springer, 61-8.

Siddiqui F, Shah SMA, Behan MY (2010). Measurement of size distribution of blasted rock using digital image processing.

Tang X (1998). Texture information in run-length matrices. IEEE T Image Process 7:1602-9.

Tessier J, Duchesne C, Bartolacci G (2007). A machine vision approach to on-line estimation of run-of-mine ore composition on conveyor belts. Miner Eng 20:1129 - 1144.

Thurley MJ (2013). Automated image segmentation and analysis of rock piles in an open-pit mine. In: Digital Image Computing: Techniques and Applications (DICTA), 2013 International Conference on. IEEE.

Wang W (1999). Image analysis of aggregates. Comput Geosci 25:71 - 81.

Wang W (2006). Image analysis of particles by modified ferret method — best-fit rectangle. Powder Technol 165:1 - 10.

Wang W (2008). Rock particle image segmentation and systems. In: Pattern Recognition Techniques, Technology and Applications. InTech.

Yaghoobi H, Mansouri H, Farsangi MAE, Nezamabadi-Pour H (2019). Determining the fragmented rock size distribution using textural feature extraction of images. Powder Technol 342:630-41.

Zhang Z, JianguoYang, Su X, Ding L, Wang Y (2013). Multi-scale image segmentation of coal piles on a belt based on the hessian matrix. Particuology $11: 549-555$. 